\title{
Detection of Sleep Apnea Based on the Analysis of Sleep Stages Data Using Single Channel EEG
}

\author{
Vijayakumar Gurrala ${ }^{1,2^{*}}$, Padmasai Yarlagadda ${ }^{1}$, Padmaraju Koppireddi $^{2}$ \\ ${ }^{1}$ Department of Electronics and Communication Engineering, VNR Vignanajyothi Institute of Engineering and Technology, \\ Hyderabad 500090, Telangana, India \\ ${ }^{2}$ Department of Electronics and Communication Engineering, JNTU Kakinada, Kakinada 533003, Andhra Pradesh, India
}

Corresponding Author Email: vijayakumar_g@vnrvjiet.in

https://doi.org/10.18280/ts.380221

Received: 10 July 2020

Accepted: 23 February 2021

\section{Keywords:}

electroencephalogram (EEG), sleep stages, sleep disorders, sleep apnea, machine learning classifiers

\begin{abstract}
Sleep is a basic need for a human being's intellectual and physiological restoration and overlaying nearly one $1 / 3$ length of a daytime. A first-rate and deep sleep is required for green regeneration of the body. Sleep disorders hamper the performance of an individual. Sleep Apnea is the one amongst the disorders that affect many. Most of Apnea related works consider Electrocardiogram (ECG) and respiratory signals /or combinations, instead of considering all Polysomnographic signals (PSG). It is evident that for the detection of Apnea related sleep disorders it is required to consider one or few signals rather considering all PSG signals. In this work, we advocate a way that might be carried out to perceive the information of sleep stages which might be crucial in diagnosing and treating sleep disorders. It differentiates sleep stages and derives new features from the sleep EEG that allows helping physicians with the analysis and treatment of associated sleep issues. This theory depends on exclusive EEG datasets from Physionet with the use of MIT-BIH polysomnographic database that have been received and described through scientists for the analysis and prognosis of sleep ranges. Experimental results on 18 records with 10197 epochs show that an Apnea detection accuracy of $95.9 \%$ obtained for Machine learning classifier with Ensemble Bagged Tree classifier.
\end{abstract}

\section{INTRODUCTION}

Sleep and mental health are closely connected and sleep deprivation affects individual's physiological and mental health condition. Sleep disorders are mainly due to lack of sleep or excessive sleep. To improve the quality of sleep, it is necessary to detect the sleep disorders at an early stage. Sleep apnea is one of the sleep disorders that everyone is suffering currently who aged around 30 . Information about brain lobes and sleep stages is necessary for the analysis of sleep disorders (apnea). Brain lobes structuring are needed to collect the Sleep EEG data from the brain locations and sleep stages data depicts the irregularity occurrence in sleep.

\subsection{Brain lobes and EEG signal recording}

The brain spatial region [1] is divided in to 4 regions of Occipital, Parietal, Temporal and Frontal cortexes shown in Figure 1. Occipital cortex is responsible for visual data processing and is used for EEG experiments related to visual stimuli. Parietal cortex is responsible for motion capabilities. Temporal cortex is responsible for speech and language processing. Frontal cortex responsible for control or monitoring our behaviour with the analysis of past activities. From these locations by affixing electrodes, sleep EEG data is collected. One can use many channels (location of electrodes at lobes) for the collection of data.

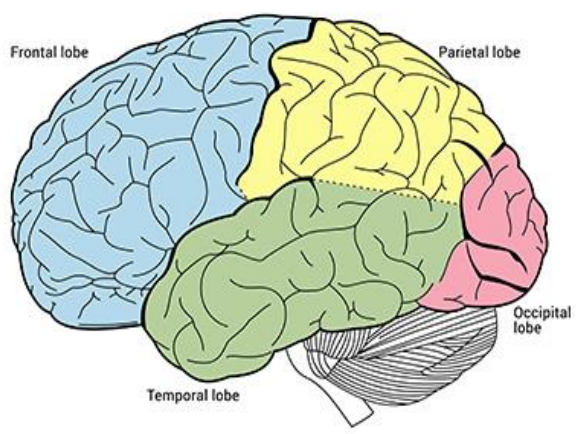

Figure 1. Brain lobes for EEG recording

EEG acquisition techniques utilize a wearable gadget characterized into the classifications of headset-based and electrode-cap based strategies. In headset type the gadget is mounted on the subject's head, and the electrode-cap uses a swimming-cap sort of acquisition gadget. In these strategies electrode-cap sort of gadget have edge over the other even the recording of EEG influenced by head movements, since it is firmly worn on the head and it has a bigger number of terminals than the headset-type gadget. The headset-type gadget has the benefits of lower cost and improved subject accommodation, since it tends to be worn effectively. The electrode cap based gadget uses 24 channels to record the data and the head set based uses less number of channel. 


\subsection{Sleep stages and disorders}

Even though we are not having consistent sleep our brain is deceptively active. There exist multiple cycles [2] it can go through with distinct brain patterns and it is important to perform normal functions when awake. It has 5 stages of sleep which occurs around 90 minutes of cycle during a normal night sleep. The first 3 stages are considered as Non-Rapid Eye Movement (NREM) split into N1, N2 and N3, then Rapid Eye Movement (REM) and lastly Wake (W) stage.

N1-It is between sleep and wakefulness. Brain starts producing theta waves and might feel hypnogogic hallucinations. These people will awake easily.

N2-This is called as deeper sleep stage. People harder to awaken. It has more theta sleep spindles (burst of rapid rhythmic brain activity) and k-complexes.

N3-This is called slow wave sleep. It has Delta wave. People difficult to wake up. Subject is very much dead to the world. Walk and Talk in sleep occurs in this stage.

REM-In this stage eyes moves rapidly beneath lids and most of the other muscles are paralyzed. Dreaming occurs in this stage. This stage is also called as paradoxical sleep. There is an active body/brain but body paralyzed.

In a normal night of uninterrupted sleep these cycles occur of 4, 5 times each and rakes about 90 minutes to complete cycles. The order it doesn't go through of 1, 2, 3 and REM. The order with in a cycle goes from N1 to N2 to N3 and then back to N2 to go to REM and then back to N1. This depends on how old are and more of NREM and REM before wakeup. Sleep problems [3] causes sleepiness, depression or may lead to improper functions of our body. People spend around one$1 / 3$ in their lives sleeping. Conditions such as Obstructive Sleep Apnea [4, 5] and insomnia are frequent and critically affect health.

\subsection{Related work}

The survey says that, 50-70 million humans suffer with sleep issues in U.S. further, more than $90 \%$ of depressive and disorder sufferers suffer from sleep issues. Sleep apnea has envisioned being common in $2 \%-4 \%$ of adults and in $1 \%-$ $3 \%$ of kids. Approximately $33 \%$ of world's population suffers from insomnia signs.

Veterans with posttraumatic stress [4] disorder had less REM and deep sleep. Nearly 1 in 3 military members who report a sleep disorder had a nightmare disorder.60\% of patients with nightmares had trauma related nightmares. Most had co morbid depression, Anxiety and Insomnia.

Kristiansen et al. [6] worked on Obstructive sleep apnea detection with the combinations of ECG-Apnea data and the respiratory signal acquired from nose and chest. The performance of the classification is testes with the data mining algorithms of artificial neural network, support vector machine, decision tree, K-nearest neighbour (KNN), and random forest and achieve $96.6 \%$ with the ECG combination and $90 \%$ with the respiratory signal acquired from nose and chest.

The researchers [7-16] worked sleep apnea detection considering different signals like ECG, respiratory with the applications different machine learning and deep learning methods and created mobile applications to get apnea score.

Most Apnea related works summarized uses ECG and/or respiratory signals combinations, instead of considering all polysomnographic signals (PSG) [17]. It is evident that for the detection of Apnea related sleep disorders it is required to consider one or few signals rather considering all PSG signals. However to the best of our knowledge Apnea detection using sleep data is not yet investigated. Whereas the literature indicates that one of the maximum essential signals is EEG and often/mostly used alerts for studying sleep staging, irrespective of category is manual or computerized. Many researchers have used more than one EEG channels. In this work we consider single channel EEG and its corresponding Sleep data.

This article is organised as section 2 provides methodology for the proposed system, section 3 explores the analysis of results obtained and applying the proposed methodology on dataset acquired from physionet database and section 4 insights the conclusion and future scope for the proposed work.

\section{METHODOLOGY}

In this work we proposed a methodology for the detection of Apnea considering data related to the sleep stages. It is a systematic step by step procedure to be followed for the detection. The whole process is given the Figure 2.

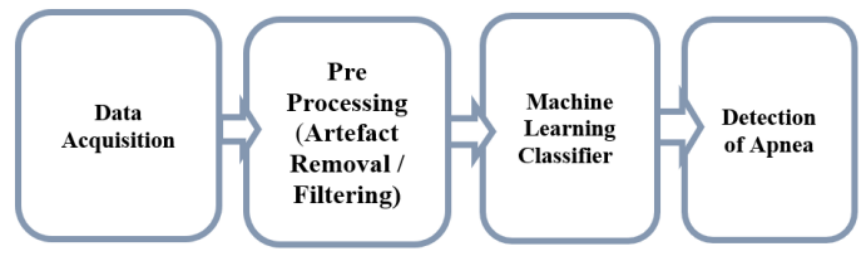

Figure 2. Block diagram of sleep apnea detection

Firstly the data has to be collected from subjects. This step is data acquisition. In this work we consider online MIT-BIH database took from physionet [18]. It is a dataset of 18 subjects, more than 80 hours of PSG data collected. This PSG is a combination of four, six and seven channels like EEG. ECG. respiratory, Blood Pressure (BP) etc. Each subjects record is associated with its ECG beat annotations, respiratory annotations and EEG sleep stages annotations for every 30 seconds of data. In this work we considered single channel EEG data and its associated sleep stage and apnea event annotations only. Since our point of interest is to design a apnea detection system with sleep stages in to the consideration. So that the recording complexity of having many sensors to be fixed on the body and the chances noise introduction will be minimised. The considered EEG signals sampling frequency is $250 \mathrm{~Hz}$ and each 30 seconds of data consists of 7500 samples in it. With the consideration of 30 seconds of epochs for each dataset, total data set consists of 10216 epochs for testing and training the designed machine learning model. So that there will be sufficient number of samples for model training and validation.

Next is the data pre-processing step. Pre-processing involves either artefact removal [19] or filtering the data. Generally during the process of acquisition the data mixed with the body movements signals called as artefacts or with the power line noise signal, since EEG signal is from $0.5 \mathrm{~Hz}$ to $100 \mathrm{~Hz}$. The removal of artefacts done by our proposed method called Complete Ensemble Empirical Decomposition with Adaptive Noise [17]. And the filtering of signal involves Wavelet Transform (WT) [20]. WT plays an important/essential role of multiresolution in the diagnostic and recognition fields. Using this transform the biomedical 
time-varying signals are compressed. It also comprises data points, into small parameters that represent a signal. Moreover, wavelet transform (WT) does not have single scale structure. It has only multiscale structure. This transform is a continuation of Orthodox Fourier transform (OFT) method. It resolves issues of non-stationary EEG signals [21]. In the wavelet transform method, the EEG signals are represented by wavelets simple and secured building blocks. The mother wavelet in WT gives rise to the wavelets as a part of functions derived through dilation and translation, that is, stretching and shifting and compression operations along the time axis.

For applying machine learning classifiers on data it has to be in the form of features. Deriving new variable(s) from existing variables/signal is known as feature creation. It helps to unleash the hidden relationship of a data set. Then with the obtained features [21, 22], a machine learning model to be trained such that it should provide a better detection of apnea. The working of machine learning [23] model is, for example, detection of spam in email service is identified in the act of classification problem [24]. This is binary class considering the fact that there are best 2 classes as spam and not spam. By utilizing some training data, the classifier will understand the class of given input variables. In this case, non-spam and spam emails which are known have to be fed as the input training data. The classifier can detect an anonymous email, when it is trained accurately. There are different types of learners.

(a) Lazy learners: Lazy learners commonly store the training data and rest until a testing data arrive. While it does, classification is achieved primarily based at the most associated data in the stored data. Lazy learners have much time to predict but less training time compared with eager learners. Ex: Case-based reasoning, k-nearest neighbour.

(b) Eager learners: Eager learner's builds up a classification model depend on the given training data prior to receive data for classification. It needs to be committing to individual hypothesis that covers the complete instance space. It takes fewer time to predict and much time to train for eager learners because the model construction. Ex: Naive Bayes, Decision Tree, Artificial Neural Networks.

The targets are additionally furnished with the train data [25] as the classification relates to supervised learning. Many domains like medical diagnosis, credit approval and target marketing, have lot of applications in classification. Eager and lazy learners are the two types of classification learners.

\section{RESULTS ANALYSIS}

In this work we consider online MIT-BIH database took from physionet of polysomnographic data. This consists of 18 records, each having 4 signals. They are of Electrocardiogram (ECG), Blood Pressure (BP), EEG and Respiratory signals. Out of which our signal of interest is EEG signal. Because the objective is to detect apnea by the analysis of sleep stages only. Each record consists of annotations of sleep stage and apnea details for each epoch. To resolve the issues of non-stationarity of EEG signal, the data is divided in to 30 seconds of sub samples. This sub division of signal is labelled as an Epoch. The Figures 3, 4 and 5 shows the examples of record (slp03) consider from the database having Y-axis label of Amplitude in milli volts and its one of the epochs. Figures 3 and 4 represents same EEG recorded signal but in specific Figure 3 presents EEG signal in samples i.e. the considered dataset consist of 720 epochs (5400000 samples) and Figure 4 presents in Hours i.e. 720 epochs of 30 seconds is 6 hours of sleep data collected. For processing the signal data by digital means, we have to get digitized signal by applying sampling process. After the application of sampling process with a sampling rate of $250 \mathrm{~Hz}$ each 30 second epoch consists of 7500 samples, we presented EEG signal in terms of samples in Figure 5.

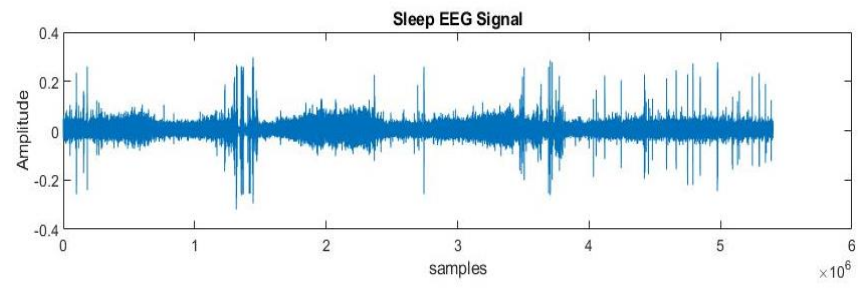

Figure 3. Sleep EEG signal in a database

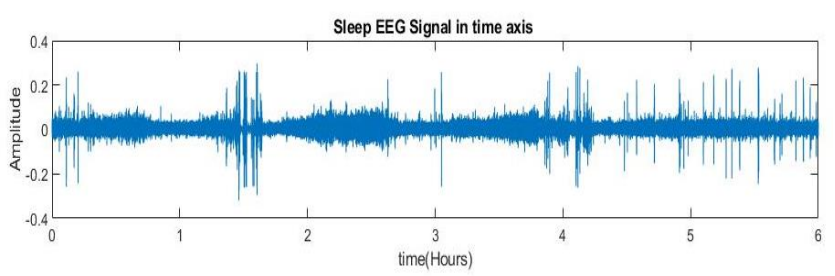

Figure 4. Sleep EEG signal in time axis

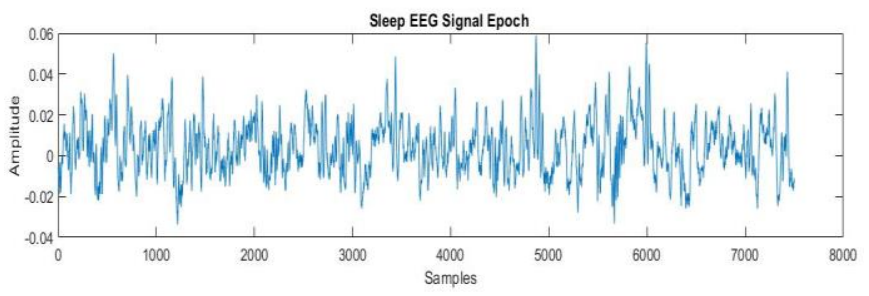

Figure 5. Sleep EEG signal epoch having 7500 samples (30 seconds)

\subsection{Feature extraction}

As an input for the classifier we will not apply directly signal epochs, instead we derive new data parameters from each epoch. These derived data called as Features. Since the processing of data through the digital means the memory requirements and the rate at which the data processing are also important factors. Hence, we consider following features from each epoch.

\subsubsection{Sleep stage (SS)}

For each epoch of 30 seconds the consideration of sleep stage gives the clarity about activity of brain signals like predominance of brain signals [26]. These stages derived from the analysis of filtered out data from WT or using the RK methods [27, 28]. The stages in sleep are Stage 1, 2, 3, R and W.

\subsubsection{Occurrence sleep stages (OSS)}

This gives the amount of occurrence of a sleep stage for each epoch in a total recorded signal. It is the ratio of how many times occurrence of particular stage to total number of stages occurred i.e. number of epochs in a considered signal. This feature determines sleep quality [29], the duration of 
sleep stages and their transition in a sleep cycle.

$$
\text { OSS }
$$$$
=\frac{\text { Amount of occurance of particular sleep stage in epoch }}{\text { Total number of sleep stages in a signal }}
$$

\subsubsection{Relative power of epoch (RPE)}

It is the ratio of power of an epoch to the sum of powers of brain signals derived from Wavelet Transform. The calculations are given below.

Power Spectral Density $(P)$ of an epoch is obtained by applying FFT on epoch $(y)$. i.e.

$$
P=\frac{\operatorname{abs}(F F T(y))^{2}}{N}
$$

where, $N$ represents number of samples in epoch.

$$
\text { Relative Power RPE }=\frac{P}{P_{\delta}+P_{\theta}+P_{\alpha}+P_{\beta}+P_{\gamma}}
$$

where, $P_{\delta}, P_{\theta}, P_{\alpha}, P_{\beta}, P_{\gamma}$ are PSDs of EEG are sub bands from epochs.

With the considered features prepare the feature vector for the machine learning classifier. The target variable of apnea data is obtained from the database. This classification is implemented on MATLAB 2020a platform. The target variables considered are No apnea $(\mathrm{N})$, Apnea (A) and Hypopnea (H) [30]. These target variables act as the labelled data for the machine learning classification model. For supervised learning implement using MATLAB, it needs a sheet with features as variable and target variables as labelled data for classification.

\subsection{Performance assessment}

It is done by considering confusion matrix and Receiver Operating Characteristics (ROC) Analysis of the classifier.

\subsubsection{Confusion matrix}

It means a desk which is frequently used to explain the overall performance of the class model on the fixed test data for which actual values are acknowledged. It is a table with four extraordinary combos of expected and real values in case for binary classifier. Confusion matrix allows us to decide mistake patterns in multi-class classification. Figure 6 shows the confusion matrix of the best machine learning classifier which detects the classes of $A, H$ and $N$.

Accuracy is given by the fraction of predictions that our model was given right. Accuracy as its own can't tell the total story when we are working with a class-imbalanced data set, in which there is a widespread disparity among the number of negative and positive labels. Recall and Precision are higher metrics for calculating class-imbalanced problems. Accuracy can be written in terms of True Positives (TP), True Negatives (TN), False Positives (FP) and False Negatives (FN) as:

$$
\text { Accuracy }=\frac{\mathrm{TP}+\mathrm{TN}}{\mathrm{TP}+\mathrm{TN}+\mathrm{FP}+\mathrm{FN}}
$$

where, TP denotes correct predictions measure for an instance to be negative; TN denotes the inappropriate predictions that an instance is positive; FP denotes the incorrect predictions measure that an instance is negative, and FN denotes the correct predictions measure for an instance to be positive.

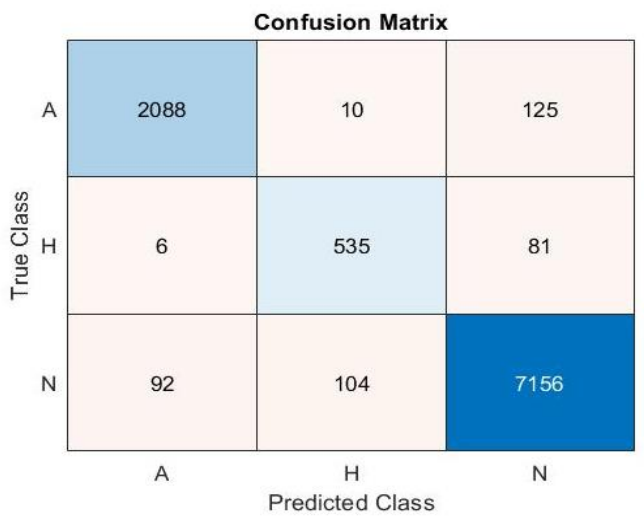

Figure 6. Confusion matrix

3.2.2 Receiver operating characteristics (ROC) analysis

The ROC space composed of combinations of possible True Positive Rate (TPR) and False Positive Rate (FPR).

$$
\begin{aligned}
& \mathrm{TPR}=\frac{\mathrm{TP}}{\mathrm{TP}+\mathrm{FN}} \\
& \mathrm{FPR}=\frac{\mathrm{FP}}{\mathrm{TN}+\mathrm{FP}}
\end{aligned}
$$

A single point in the ROC space is determined by one TPR and one FPR together, and the location of a point in the ROC space indicates the trade-off between sensitivity and specificity, i.e. the rise in sensitivity is followed by a decrease in specificity. The location of the point in the ROC space thus illustrates whether the classification diagnostic is good or not. In a best classification scenario the ROC curve is locate a point at left upper corner i.e. $(0,1)$. Figure 7 shows the ROC plots of the individual classes $\mathrm{A}, \mathrm{H}$ and $\mathrm{N}$. From the figures 7 (a), (b) and (c) the classifier's ROC is almost nearer to the left upper corner. It depicts that with the considered features the classifier gives best classification for the detection of apnea events/classes of A, $\mathrm{H}$ and $\mathrm{N}$.

From the Table 1, it is observed that Ensemble [31] Bagged Tree learner has the highest accuracy of $95.9 \%$. Ensemble learning model combine different learners results to get improved results. It is done by methods of voting or averaging. For classification Voting is applied and for regression averaging will be applied. Voting is the process of collecting prediction (voting) results for each test instance and the final prediction can consider more than half of votes, where as in averaging for each instance average predication is taken into the consideration. The objective here is to produce better performance with the ensembles of learners rather with single learners. Like Bagging, Boosting and stacking there exists 3 types of algorithms in ensemble learners. Ensemble algorithms boosts accuracy of the consider dataset. Once the model is trained with the dataset, this model can be used to predict apnea on new dataset.

Table 1. Classification accuracy comparison for the proposed method

\begin{tabular}{ccc}
\hline S.No & Classifier & Accuracy (\%) \\
\hline 1 & Tree & 95.1 \\
2 & Naïve Bayes & 76.6 \\
3 & SVM & 81.1 \\
4 & KNN & 94.9 \\
5 & Ensemble Bagged Tree & $\mathbf{9 5 . 9}$ \\
\hline
\end{tabular}




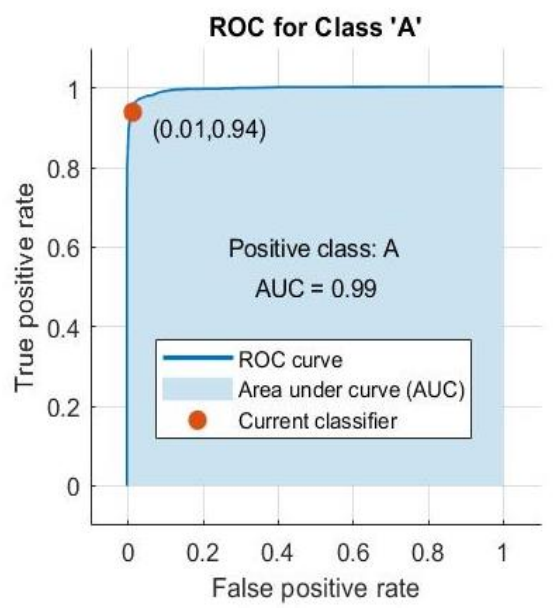

(a)

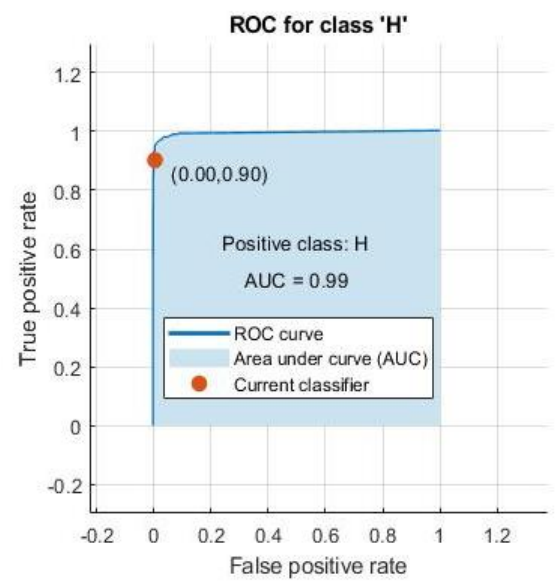

(b)

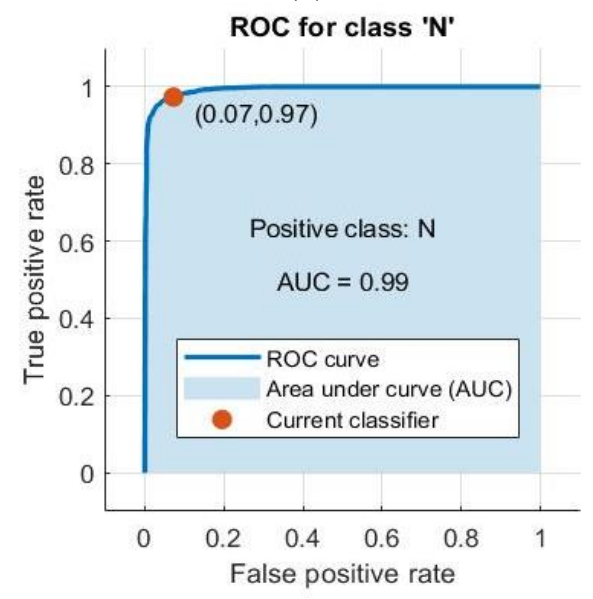

(c)

Figure 7. ROC plots for the classes A, H and N

\section{CONCLUSION AND FUTURE SCOPE}

In this work we consider features derived from EEG signal only and the experimental Machine Learning Classifier results better compared to earlier methods which includes ECG, BP, respiratory signals etc. Since the signal of interest is EEG signal only the computational complexity of the proposed method is low when compared to the earlier methods. The proposed method of Apnea detection tested on physionet polysomnographic database consisting of 10197 epochs achieves an accuracy of $95.9 \%$ with Ensemble Bagged Tree classifier. With the consideration less number of features like sleep stage information, occurrence of sleep stages and relative power makes the model simple and the considered few features uniquely represents the data in compact and the detection of sleep disorders done with the analysis of sleep stages only. The proposed Ensemble learning classifier model combine different learners results to get improved results. The occurrences of majority number of epoch samples in the diagonal of confusion matrix shows the high accuracy of the proposed model. Only EEG signal consideration decrease the process time and memory requirements also. It would be useful to design a model/device which can automatically detects apnea and gives apnea score. So that it reduces the workload of a physician and hence the decision making of a Neuro physician can be simplified to diagnose the sleep apnea. Once if we could able to detect how many times the occurrence of apnea events like A,H and $\mathrm{N}$ then we can define AHI of the particular subject. Further it is suggested that an IOT based data retrieval systems will provide ease of accessing the data for the physicians from the subjects.

\section{ACKNOWLEDGMENT}

This work has been funded by University Grants Commissions-South Eastern Regional Office (UGC-SERO) under Minor Research Project number 6620/16.

\section{REFERENCES}

[1] Lobes of Brain. [Online]. Available: https:/qbi.uq.edu.au/brain/brain-anatomy/lobes-brain, accessed on 10 Jan 2020.

[2] Khalighi, S., Sousa, T., Pires, G., Nunes, U. (2013). Automatic sleep staging: A computer assisted approach for optimal combination of features and polysomnographic channels. Expert Systems with Applications, $\quad 40(17)$ : 7046-7059. https://doi.org/10.1016/j.eswa.2013.06.023

[3] Sleep disorders. [Online]. Available: https://www.cdc.gov/sleep/about_sleep/key_disorders.h tml, accessed on 10 Jan 2020.

[4] Sleep Apnea. [Online]. Available: https://www.mayoclinic.org/diseases-conditions/sleepapnea/symptoms-causes/syc-20377631, accessed on 10 Jan 2020

[5] Alqassim, S., Ganesh, M., Khoja, S., Zaidi, M., Aloul, F., Sagahyroon, A. (2012). Sleep apnea monitoring using mobile phones. In 2012 IEEE 14th International Conference on e-Health Networking, Applications and Services (Healthcom), pp. 443-446. https://doi.org/10.1109/HealthCom.2012.6379457

[6] Kristiansen, S., Hugaas, M. S., Goebel, V., Plagemann, T., Nikolaidis, K., Liestøl, K. (2018). Data mining for patient friendly apnea detection. IEEE Access, 6: 7459874615. https://doi.org/10.1109/ACCESS.2018.2882270

[7] Behar, J., Roebuck, A., Shahid, M., Daly, J., Hallack, A., Palmius, N., Stradling, J., Clifford, G.D. (2014). SleepAp an automated obstructive sleep apnoea screening application for smartphones. IEEE Journal of Biomedical and Health Informatics, 19(1): 325-331. https://doi.org/10.1109/JBHI.2014.2307913

[8] Yadollahi, A., Moussavi, Z. (2009). Acoustic obstructive 
sleep apnea detection. In 2009 Annual International Conference of the IEEE Engineering in Medicine and Biology Society, Minneapolis, MN, USA, pp. 7110-7113. https://doi.org/10.1109/IEMBS.2009.5332870

[9] Camc1, B., Kahveci, A.Y., Arnrich, B., Ersoy, C. (2017). Sleep apnea detection via smart phones. In 2017 25th Signal Processing and Communications Applications Conference (SIU), Antalya, Turkey, pp. 1-4. https://doi.org/10.1109/SIU.2017.7960484

[10] Ward, S.L.D., Amin, R., Arens, R., Chen, Z., Davis, S., Gutmark, E., Superfine, R., Wong, B., Zdanski, C., Khoo, M.C. (2014). Pediatric sleep-related breathing disorders: advances in imaging and computational modeling. IEEE Pulse, 5(5): 33-39. https://doi.org/10.1109/MPUL.2014.2339293

[11] Jane, R. (2014). Engineering sleep disorders: From classical CPAP devices toward new intelligent adaptive ventilatory therapy. IEEE Pulse, 5(5): 29-32. https://doi.org/10.1109/MPUL.2014.2339292

[12] Hwang, S.H., Lee, Y.J., Jeong, D.U., Park, K.S. (2016). Apnea-hypopnea index prediction using electrocardiogram acquired during the sleep-onset period. IEEE Transactions on Biomedical Engineering, 64(2): 295-301. https://doi.org/10.1109/TBME.2016.2554138

[13] Leong, W.Y. (2014). Feature extraction and optimisation for sleep apnea. In 2014 IEEE International Symposium on Robotics and Manufacturing Automation (ROMA), pp.

200-205. https://doi.org/10.1109/ROMA.2014.7295888

[14] Nemati, S., Orr, J., Malhotra, A. (2014). Data-driven phenotyping: Graphical models for model-based phenotyping of sleep apnea. IEEE Pulse, 5(5): 45-48. https://doi.org/10.1109/MPUL.2014.2339402

[15] Shahin, M., Ahmed, B., Hamida, S.T.B., Mulaffer, F.L., Glos, M., Penzel, T. (2017). Deep learning and insomnia: Assisting clinicians with their diagnosis. IEEE Journal of Biomedical and Health Informatics, 21(6): 1546-1553. https://doi.org/10.1109/JBHI.2017.2650199

[16] Thanuja, R., Balakrishnan, R. (2013). Real time sleep apnea monitor using ECG. In 2013 IEEE Conference on Information \& Communication Technologies, Thuckalay, India, pp. 973-976. https://doi.org/10.1109/CICT.2013.6558237

[17] Schätz, M., Procházka, A., Kuchyňka, J., Vyšata, O. (2020). Sleep apnea detection with polysomnography and depth sensors. Sensors, 20(5): 1360. https://doi.org/10.3390/s20051360

[18] Goldberger, A.L., Amaral, L.A., Glass, L., Hausdorff, J.M., Ivanov, P.C., Mark, R.G., Mietus, G.E., Moody, G.B., Peng, C.K., Stanley, H.E. (2000). PhysioBank, PhysioToolkit, and PhysioNet: Components of a new research resource for complex physiologic signals. Circulation, 101(23): e215-e220. https://doi.org/10.1161/01.CIR.101.23.e215

[19] Gurrala, V., Yarlagadda, P., Koppireddi, P. (2017). Artifact removal from sleep hipnograms using complete ensemble empirical decomposition with adaptive noise. Indian Journal of Science and Technology, 10: 1-8. https://doi.org/10.17485/ijst/2017/v10i47/108645

[20] Guo, C., Yu, J., Wu, L., Liu, Y., Jia, C., Xie, Y. (2019). Analysis and feature extraction of EEG signals induced by anesthesia monitoring based on wavelet transform. IEEE Access, 7: 41565-41575. https://doi.org/10.1109/ACCESS.2019.2907794

[21] Gurrala, V., Yarlagadda, P., Koppireddi, P. (2019). Wavelet domain extraction of features from single channel sleep EEG. International Journal of Innovative Technology and Exploring Engineering (IJITEE), 8(4S2).

[22] Delimayanti, M.K., Purnama, B., Nguyen, N.G., Faisal, M.R., Mahmudah, K.R., Indriani, F., Kubo, M., Satou, K. (2020). Classification of brainwaves for sleep stages by high-dimensional FFT features from EEG signals. $\begin{array}{lll}\text { Applied } \quad \text { Sciences, } & 1797 .\end{array}$ https://doi.org/10.3390/app10051797

[23] Khare, A., Jeon, M., Sethi, I.K., Xu, B. (2017). Machine learning theory and applications for healthcare. Journal of Healthcare Engineering, 2017: 5263570. https://doi.org/10.1155/2017/5263570

[24] Sang, S., Yang, Z., Li, Z., Lin, H. (2015). Supervised learning based hypothesis generation from biomedical literature. BioMed Research International, 2015: 1-12. https://doi.org/10.1155/2015/698527

[25] Hunter, J. (2002). Biomedical Datasets. Agenda.

[26] Längkvist, M., Karlsson, L., Loutfi, A. (2012). Sleep stage classification using unsupervised feature learning. Advances in Artificial Neural Systems, 2012: 1-9. https://doi.org/10.1155/2012/107046

[27] Novelli, L., Ferri, R., Bruni, O. (2010). Sleep classification according to AASM and Rechtschaffen and Kales: Effects on sleep scoring parameters of children and adolescents. Journal of Sleep Research, 19(1p2): 238-247. 2869.2009.00785.x https://doi.org/10.1111/j.1365-

[28] Sleep Computing Committee of the Japanese Society of Sleep Research Society (JSSR), Hori, T., Sugita, Y., Koga, E., Shirakawa, S., Inoue, K., Uchida, S., Kuwahara, H., Kousaka, M., Kobayashi, T., Tsuji, Y., Terashima, M., Fukuda, K., Fukuda, N. (2001). Proposed supplements and amendments to 'A manual of standardized terminology, techniques and scoring system for sleep stages of human subjects', the Rechtschaffen \& Kales (1968) standard. Psychiatry and Clinical Neurosciences, 55(3): 305-310. https://doi.org/10.1046/j.1440-1819.2001.00810.x

[29] Libman, E., Fichten, C., Creti, L., Conrod, K., Tran, D. L., Grad, R., Bailes, S. (2016). Refreshing sleep and sleep continuity determine perceived sleep quality. Sleep Disorders, 2016: 7170610. https://doi.org/10.1155/2016/7170610

[30] Alchanatis, M., MacFarlane, J., Schiza, S. (2012). Sleep Apnea. Sleep Disord., 2012: 308978. https://doi.org/10.1155/2012/308978

[31] Jin, L.P., Dong, J. (2016). Ensemble deep learning for biomedical time series classification. Computational Intelligence and Neuroscience, 2016: 1-13. https://doi.org/10.1155/2016/6212684 